\title{
Capacitação em antropometria como base para o fortalecimento do Sistema de Vigilância Alimentar e Nutricional no Brasil
}

\author{
Anthropometry training as basis for \\ strengthening the Brazilian Food and \\ Nutritional Surveillance System
}

Ursula Viana BAGNI

Denise Cavalcante de BARROS²

\section{R E S U M O}

\section{Objetivo}

Apresentar recomendações acerca do planejamento de capacitações em antropometria em âmbito local na Atenção Primária à Saúde, a fim de subsidiar a geração de informações nutricionais de boa qualidade e fortalecer a vigilância nutricional.

\section{Métodos}

Diante da ausência de documentos oficiais que normatizem periodicidade, metodologia, conteúdo e outros aspectos relevantes ao adequado planejamento e execução de capacitações em antropometria em âmbito local, as recomendações apresentadas são baseadas em experiências bem-sucedidas de um Laboratório de Antropometria com vocação para formação de recursos humanos, pertencente a uma instituição federal do Rio de Janeiro.

\section{Resultados}

Reciclagens periódicas devem integrar de forma permanente a agenda dos serviços de saúde, sendo desenvolvidas ao menos uma vez por semestre, em pequenos grupos. Os equipamentos antropométricos devem estar disponíveis em todos os momentos, e para uma capacitação abordando peso, estatura e perímetro da cintura, deve haver, no mínimo, 1 balança, 1 antropômetro e 2 fitas antropométricas para cada 6 participantes. Uma capacitação contemplando até 3 medidas corporais deve ter carga horária mínima de 4 horas, sendo metade direcionada para sensibilização, troca de experiências e revisão das técnicas, e o restante para treinamento, identificação e correção de erros, avaliação da qualidade das medidas e discussão dos resultados. Essas atividades devem se constituir em espaço de troca de experiências que favoreça o aperfeiçoamento das técnicas consi-

\footnotetext{
${ }^{1}$ Universidade Federal do Rio Grande do Norte, Faculdade de Ciências da Saúde do Trairi. R. Trairi, s/n., Centro, 59200-000,

Santa Cruz, RN, Brasil. Correspondência para/Correspondence to: UV BAGNI. E-mail: <ursulaviana@gmail.com>.

2 Fundação Oswaldo Cruz, Escola Nacional de Saúde Pública, Centro Colaborador em Alimentação e Nutrição da Região Sudeste. Rio de Janeiro, RJ, Brasil.
} 
394 | UV BAGNI \& DC BARROS

derando o contexto de atuação e dificuldades enfrentadas pelos profissionais em sua rotina. Deve-se dar ênfase na prática supervisionada das medições, pois permitem identificar e corrigir erros, assim como avaliar a precisão e exatidão dos profissionais.

\section{Conclusão}

O aperfeiçoamento e uniformização das capacitações em antropometria poderão favorecer o aprendizado e desenvolvimento do profissional, bem como garantir o alcance dos objetivos de promoção e vigilância em saúde do Sistema Único de Saúde.

Termos de indexação: Antropometria. Capacitação em serviço. Serviços de Saúde. Vigilância Nutricional.

\section{A B S T R A C T}

\section{Objective}

This study provides recommendations for planning anthropometry training in primary healthcare to improve nutrition information quality and strengthen nutrition surveillance.

\section{Methods}

Since there are no official documents establishing a periodicity, methodology, content and other relevant aspects for planning and performing local anthropometry training, this manuscript offers recommendations based on the successful experiences of an anthropometric laboratory skilled in training human resources. The laboratory belongs to a federal institution in Rio de Janeiro.

\section{Results}

Refresher courses should be included in the health service schedule and offered at least once per semester for small groups. Anthropometric devices should be available at all times. A training course involving weight, height and waist circumference measurements should contain at least 1 scale, 1 stadiometer and 2 anthropometric tapes for every 6 trainees. A course including 3 body measurements should last at least 4 hours, 2 dedicated to increasing awareness, exchange of experiences and reviewing techniques and 2 dedicated to training, error identification and correction, assessment of measurement quality and discussion of the results. These activities should constitute a space for the exchange of experiences that improve techniques, considering the context of the trainees' work and the difficulties they face daily at work. Emphasis should be given to supervised measurement collection, since it allows the identification and correction of errors and assessment of the trainees' precision and accuracy.

\section{Conclusion}

Improvement and standardization of anthropometry training can promote learning and professional development, and ensure the accomplishment of the Unified Healthcare System's objectives, which are to promote and surveil health.

Indexing terms: Anthropometry. Inservice Training. Health services. Nutritional surveillance.

\section{N T R O D U Ç Ã O}

A antropometria é uma importante ferramenta para a avaliação do estado nutricional, e os indicadores antropométricos gerados a partir das medidas corporais são importantes preditores das condições de saúde e sobrevida de indivíduos e populações ${ }^{1,2}$.

Atendendo à terceira diretriz da Política Nacional de Alimentação e Nutrição (PNAN)³ ${ }^{3}$ que trata da avaliação e monitoramento da situação alimentar e nutricional da população brasileira, o
Sistema de Vigilância Alimentar e Nutricional (SISVAN) vem sendo implementado no Brasil para fornecer continuamente informações sobre as condições nutricionais da população atendida pelo Sistema Único de Saúde (SUS) de todo o país. São coletadas informações de peso e estatura de crianças, adolescentes, adultos, idosos e gestantes, e mais recentemente a medida do perímetro da cintura em adultos, por ser um indicador importante do risco cardiovascular ${ }^{4}$.

Segundo Barros et al. ${ }^{5}$, "a antropometria não deve ser entendida apenas como uma simples 
ação de pesar e medir, mas, sobretudo, como uma atitude de vigilância, isto é, um olhar atento para o estado nutricional, permitindo uma intervenção precoce, quando verificada alguma alteração" (p.15). Em âmbito coletivo, é considerada fundamental para apoiar as ações de assistência e promoção à saúde, assim como para nortear a formulação e aperfeiçoamento de políticas públicas de prevenção, detecção e controle de agravos nutricionais ${ }^{4}$, ficando clara a importância da coleta criteriosa e padronizada das medidas corporais em todas as unidades de saúde do país, baseadas nas técnicas recomendadas pelo governo federal.

No intuito de apoiar o SISVAN no País, a Coordenação-Geral de Alimentação e Nutrição (CGAN) do Ministério da Saúde desenvolveu, no ano de 2005, onze Capacitações Macrorregionais em Vigilância Alimentar e Nutricional para os profissionais da atenção primária à saúde, com a colaboração dos Centros Colaboradores de Alimentação e Nutrição (CECAN) e pesquisadores de universidades. Tais eventos resultaram na publicação de um relatório com a metodologia utilizada a fim de permitir sua implantação e/ou implementação pelos recursos humanos na esfera municipal, segundo a realidade de cada local ${ }^{6}$.

A CGAN também elaborou e distribuiu materiais de apoio técnico para todos os municípios e regionais de saúde, universidades de Nutrição, CECAN e Áreas Técnicas Estaduais de Alimentação e Nutrição, de modo a esclarecer os objetivos e normalizar os parâmetros estabelecidos pelo SISVAN, tais como manuais técnicos com orientações básicas para a coleta, o processamento, a análise de dados e a informação em serviços de saúde, cartazes, álbuns seriados de antropometria, álbuns seriados sobre alimentação saudável, dentre outros ${ }^{6}$.

Ainda não foram publicados, todavia, documentos oficiais que abordem questões fundamentais para o planejamento e a execução de reciclagens e capacitações periódicas em antropometria para profissionais da atenção primária à saúde em âmbito local, isto é, nos serviços de saúde, particularmente quanto à sua periodi- cidade, número de participantes, carga horária teórico-prática, conteúdo a ser abordado, metodologia e avaliação do aprendizado.

A ausência de tais diretrizes tem resultado em uma gama de atividades malsucedidas, desenvolvidas de forma isolada, despadronizada e repetitiva, e também com pouco tempo direcionado à prática das medições corporais, o que pouco favorece o aperfeiçoamento do profissional e desestimula sua participação. Além disso, a falta de uniformidade e de sistematização quanto à periodicidade e continuidade entre os treinamentos já realizados tem prejudicando a avaliação da evolução do profissional antropometrista em médio e longo prazo, dificultando a consecução dos objetivos propostos.

Melhorar a qualidade das capacitações em antropometria direcionadas aos profissionais do SUS certamente pode fortalecer o SISVAN, na medida em que as informações coletadas se tornam mais fidedignas e robustas para orientar de forma apropriada as ações em saúde pública.

Indo ao encontro dessas necessidades, este artigo apresenta recomendações e sugestões acerca do planejamento de capacitações em antropometria na atenção básica, baseadas nas vivências e experiências bem-sucedidas de um laboratório de antropometria situado em uma instituição pública federal de ensino e pesquisa do Rio de Janeiro. Com vocação na área de formação de recursos humanos, esse laboratório já capacitou centenas de profissionais de saúde, e tem apoiado a Coordenação-Geral de Alimentação e Nutrição do Ministério da Saúde na formação de recursos humanos em toda a região Sudeste do país.

\section{A importância da padronização das medidas antropométricas para a epidemiologia nutricional}

Para que as medidas antropométricas reflitam com segurança o estado nutricional, é necessário que o profissional seja preciso e exato em 
sua mensuração. A precisão (ou reprodutibilidade) está relacionada à capacidade de o profissional obter valores semelhantes (ou bem próximos) em medições repetidas no mesmo indivíduo, enquanto a exatidão (ou validade) se refere à habilidade de obter valores similares ao real, encontrado por um antropometrista com larga experiência ${ }^{7,8}$. Medidas coletadas por profissionais com baixa precisão e/ou exatidão distorcem a estimativa dos desvios nutricionais, o que traz prejuízos tanto para o indivíduo quanto para a coletividade. A subestimativa de deficits e excessos nutricionais favorece a perpetuação e agravamento desses distúrbios, uma vez que os indivíduos doentes são privados de um atendimento mais individualizado. Além disso, medidas públicas de intervenção deixam de ser aprimoradas ou até mesmo implementadas por falta de dados concretos sobre o problema. Em contrapartida, a superestimativa dos agravos implica má utilização dos recursos de programas assistenciais, uma vez que intervenções são realizadas desnecessariamente em indivíduos sadios, trazendo sobrecarrega aos serviços de saúde ${ }^{5,9}$.

Antropometristas que pouco reciclam seus conhecimentos estão sujeitos a cometer uma série de erros de medição, tanto aleatórios quanto sistemáticos $2,10,11$. Bagni et al. ${ }^{9}$ demonstraram que quanto menor a prática e o treinamento prévio do antropometrista, maior a ocorrência de erros nas medições, resultando em baixa precisão e exatidão, constatando a extrema importância das capacitações para os profissionais de saúde.

Programas periódicos de formação profissional em antropometria melhoram sobremaneira a qualidade das medidas coletadas pelo profissional, favorecendo um diagnóstico nutricional fidedigno ${ }^{10,12}$. Nos serviços de saúde, essa atividade favorece não somente a identificação e a correção de erros de medidas de antropometristas iniciantes, mas também ajuda na manutenção da qualidade das mensurações dos profissionais já capacitados que atuam rotineiramente nessa atividade ${ }^{10}$, garantindo uma equipe preparada e comprometida com a oferta de um serviço de qualidade à população.

\section{Programa de formação profissional em antropometria: treinamento ou capacitação?}

O principal patrimônio das organizações são as pessoas: são elas que prestam serviços, conduzem atividades, elaboram produtos. Mas para que essas atividades sejam realizadas de forma bem-sucedida, é imprescindível o treinamento e o desenvolvimento das pessoas por meio de programas de formação profissional ${ }^{13}$. Nesse contexto, é importante perceber que, apesar de ambos os termos treinamento e capacitação constituírem processos de aprendizagem, eles não são sinônimos e denotam processos distintos.

Treinar, de acordo com o dicionário da língua portuguesa ${ }^{14}$, é sinônimo de exercitar, adestrar, habituar, acostumar. Segundo Chiavenato ${ }^{13}$, o treinamento profissional é "o processo pelo qual a pessoa é preparada para desempenhar de maneira excelente as tarefas específicas do cargo que deve ocupar" (p.339), desenvolvendo suas competências para que se tornem mais produtivas, criativas e inovadoras. Para Chiavenato ${ }^{13}$, o foco dos programas de treinamento está em transmitir informações, diretrizes, regras e procedimentos, assim como desenvolver habilidades, novos hábitos e atitudes, tornando os profissionais mais eficazes em suas tarefas.

Já o termo capacitar é definido pelo dicionário como sinônimo de habilitar, fazer capaz, convencer, persuadir ${ }^{14}$. Para Chiavenato $^{13}$, em se tratando de gestão de pessoas, a capacitação é um processo de educação e orientação para o futuro, que busca o desenvolvimento e o crescimento pessoal dos profissionais que dele participam.

Chiavenato $^{13}$ ressalta que, embora o treinamento e o desenvolvimento de pessoas afetem de maneira similar a aprendizagem, a perspectiva de tempo de cada um é diferente. Segundo Chiavenato ${ }^{13}$, "o treinamento é orientado para o presente", visando aperfeiçoar a maneira como o profissional desempenha suas atividades. Já a capacitação está mais relacionada 
a "processos mais profundos de formação da personalidade e da melhoria da capacidade de compreender e interpretar o conhecimento, do que com a repetição de um conjunto de fatos e informações a respeito das habilidades motoras ou executoras" (p.370).

Nesse contexto, fica claro que, em se tratando de antropometria, ambas as atividades estão indissociavelmente interligadas e constituem o que aqui será considerado um programa de formação profissional em antropometria. Treinamentos práticos devem ser realizados de forma contínua, constante e ininterrupta, mesmo quando os profissionais apresentam bom desempenho, já que a participação nessas atividades incentiva a melhoria contínua e a busca de patamares mais elevados de desempenho ${ }^{13}$. Entretanto, mais do que simplesmente treinar técnicas de medição, é necessário que haja um trabalho constante de sensibilização quanto à importância do cuidado durante a coleta dessas medidas, a fim de fortalecer a cultura organizacional e garantir que os objetivos de promoção, atenção e vigilância em saúde do SUS sejam alcançados.

Dessa maneira, a capacitação em antropometria, termo que neste artigo denominará a atividade conjugada de sensibilização e treinamento dentro de um programa de formação profissional, não deve ser vista como um evento isolado, ou mais uma atividade de que os profissionais simplesmente "precisam participar"15, mas sim como uma oportunidade para reforçar bons hábitos de trabalho e impulsionar a organização em uma trajetória ascendente.

\section{Desenvolvendo capacitações em antropometria}

Um dos principais pré-requisitos para que capacitações sejam bem-sucedidas é que sejam valorizadas por todos os profissionais da instituição, desde os gestores até os trabalhadores, sendo essencial que se crie e desenvolva uma cultura interna favorável ao aprendizado. A organização precisa estar disposta a dar espaço e oferecer oportunidades para que as pessoas possam aplicar as novas competências e conhecimentos adquiridos na capacitação ${ }^{13}$.

Os processos de desenvolvimento de pessoas vêm tradicionalmente seguindo o modelo casual (apenas quando surge necessidade ou oportunidade), em uma atitude reativa (apenas quando existe um problema ou necessidade), em uma visão de curto prazo (visando apenas ao problema imediato), em esquema randômico (as pessoas são escolhidas aleatoriamente, e nem todas participam), baseado na imposição (as pessoas não são consultadas), em uma condição de estabilidade e conservação (nada realmente muda após a capacitação) e visando ao permanente e definitivo ${ }^{13}$. Chiavenato ${ }^{13}$ alerta, contudo, que se deve buscar uma abordagem mais moderna para as capacitações, migrando para um modelo planejado (capacitação como parte da cultura), em uma atitude proativa (antecipando-se as necessidades), em uma visão de longo prazo (visando ao futuro), em um esquema intencional (todas as pessoas participam), baseado no consenso (as pessoas são consultadas e convidadas), em uma condição de instabilidade, mudança, inovação e criatividade (buscando sempre mudar para melhor) e visando ao provisório e mutável.

Nessa mesma linha, Freire ${ }^{16}$, consagrado por desenvolver uma pedagogia que discute a contradição "opressores-oprimidos", valendo-se do método materialista-dialético de análise da realidade, propõe uma educação problematizadora, que visa, em última instância, à práxis do diálogo (dialogicidade) como um importante princípio educativo. Freire ${ }^{16}$, considera que "o diálogo é esse encontro dos homens, mediatizados pelo mundo, e perpassa todo o processo de ensino-aprendizagem, começando na busca do conteúdo programático e se estendendo até as avaliações finais do processo" (p.78).

As capacitações devem, assim, adotar um método pedagógico relativamente simples, que estruture um processo de ensino-aprendizagem em acordo com o contexto vivido pelos participantes. Essa opção em trabalhar com conteúdos 
presentes no dia a dia do grupo visa a provocar nesses profissionais de saúde não apenas uma identificação com o que está sendo estudado, mas (e principalmente) o exercício do questionamento e a problematização da sua realidade. Esse método busca associar dois objetivos interligados: a instrumentalização teórico-prática dos participantes, que querem a apropriação do conhecimento -, e um posicionamento progressivamente crítico e questionador, possibilitando uma ressignificação de posturas e valores reprodutores de uma estrutura social vigente no seu contexto de vida ${ }^{16}$. Refere-se aqui a um profissional com posicionamento e ação críticos diante de uma realidade compreendida a partir de um processo contínuo de reflexão e questionamento (consciência).

Capacitações devem ser cíclicas e ter continuidade, sendo geralmente compostas por quatro etapas que se complementam: diagnóstico, planejamento, implementação e avaliação. A primeira etapa, que compreende o diagnóstico, é quando são levantadas as necessidades de treinamento a serem satisfeitas, as quais podem ser passadas, presentes ou futuras. Nem sempre todas as necessidades são claras, e podem ser necessários levantamentos e pesquisas internas para identificar os descompassos entre o que deveria ser feito e o que realmente é realizado ${ }^{13}$. Em antropometria, o diagnóstico pode ser feito tanto pela avaliação periódica de prontuários e do sistema de informações para identificação de dados inconsistentes e/ou incoerentes (por exemplo: indivíduos que "encolheram" de uma consulta para outra), quanto pela observação da aplicação das técnicas e procedimentos pelos profissionais durante os atendimentos aos clientes ou treinamentos periódicos.

Na prática, identificar problemas relacionados às técnicas de medição é muito difícil, pois quando os profissionais sabem que estão sendo observados, eles tendem a alterar seu comportamento, intensificando o cuidado em suas medições a fim de evitar a constatação de possíveis falhas em sua atuação. Além disso, erros e in- coerências em prontuários não permitem identificar a fonte do erro, isto é, se o equívoco ocorreu realmente durante a mensuração (técnica incorreta, falta de atenção, pressa) ou se foi no momento do registro no formulário (anotação equivocada, letra ilegível), da digitação ou em alguma outra etapa. Por esse motivo, recomenda-se que a revisão das técnicas antropométricas esteja permanentemente no rol de atividades das capacitações, devendo ser incorporada à programação oficial dos serviços de saúde, independentemente de os profissionais executarem satisfatoriamente a tarefa ou mesmo de outras necessidades terem sido identificadas.

A segunda etapa é a elaboração do desenho do programa de capacitação para atender as necessidades diagnosticadas, devendo contemplar: (1) quem deve ser capacitado (treinandos); (2) conteúdo ou assunto a ser apresentado; (3) como deve ser conduzida a capacitação (métodos e recursos instrucionais); (4) quem deve ministrar a capacitação (instrutor); (5) local onde será realizada a capacitação e (6) quando será executada a atividade (época, periodicidade, horário do treinamento) ${ }^{13}$.

Recomenda-se que todos os profissionais devam ser capacitados a fim de exercerem a função de antropometristas. Para aqueles que já trabalham nessa atividade em sua rotina (nutricionistas, médicos, enfermeiros, técnicos de enfermagem, agentes comunitários de saúde e outros), as capacitações devem ocorrer, no mínimo, uma vez por semestre. Contudo, nas unidades onde há maior rotatividade de profissionais, essas atividades devem ser realizadas com maior frequência. Dessa forma, é possível manter a equipe sensibilizada quanto ao seu papel para o correto diagnóstico nutricional; aperfeiçoar as técnicas de medição e de manuseio dos equipamentos, corrigindo erros e vícios de trabalho, de modo a ter uma equipe bem treinada e padronizada; preparar os profissionais para lidar sabiamente com situações não usuais, padronizando os procedimentos a serem adotados quando surgirem dificuldades de medição, a fim de evitar erros futuros. 
É importante que o convite para a participação nessa atividade seja feito de forma adequada, de modo que o profissional se sinta valorizado, e não cobrado ou criticado. Ao se propor uma atividade de capacitação em técnicas antropométricas no serviço, deve-se ressaltar o conceito de melhoria contínua e de busca pela excelência, e enfatizar o fato de a presença dos antropometristas ser um investimento da unidade nesses profissionais por se considerá-los "peças-chave" no serviço, afastando assim qualquer conotação de que foram convidados em decorrência de má qualidade do trabalho.

As capacitações periódicas devem ser estruturadas de modo a contemplar minimamente os seguintes pontos: 1) sensibilização quanto à importância do cuidado durante a coleta das medidas antropométricas para o correto diagnóstico nutricional individual e coletivo; 2) revisão das técnicas corretas de medição (o número de medidas a ser abordado poderá variar conforme as especificidades dos serviços de saúde, sendo as mais usadas na atenção primária o peso, a estatura e o perímetro da cintura) e 3) prática das medidas antropométricas. O roteiro das capacitações, contudo, pode ser flexível, devendo ser ajustado aos participantes e às necessidades identificadas pela instituição ${ }^{15}$.

As capacitações devem se configurar numa troca de experiências, em que, mais do que transmitir informações e reforçar atitudes positivas $^{15}$, seja possível conhecer a diversidade de vivências e dificuldades enfrentadas pela equipe em sua rotina, assim como suas dúvidas, opiniões e sugestões para melhorar o serviço.

Para favorecer a participação ativa de todos os profissionais nas discussões em grupo, assim como a organização e supervisão da prática das técnicas de medição, recomenda-se que as capacitações não excedam 12 participantes. Como recursos didáticos, podem ser realizadas aulas expositivas com vídeos, cartazes, álbuns seriados, discussões em grupo, práticas observadas, dentre outros. Formulários e prontuários da unidade, caderneta de saúde da criança, calculadoras, tabe- las de referência e disco para cálculo do índice de massa corporal podem ser utilizados como material de apoio durante as atividades. Na página eletrônica da Coordenação-Geral de Alimentação e Nutrição do Ministério da Saúde (www.saude. gov.br/nutricao), estão disponíveis diversos materiais que podem ser usados para capacitação em antropometria nas unidades de saúde da atenção básica. Os equipamentos antropométricos devem estar disponíveis em todos os momentos da atividade, e para um grupo de 12 profissionais da atenção básica realizando treinamento sobre peso, estatura e perímetro da cintura, deve-se ter disponível, no mínimo, 2 balanças, 2 antropômetros e 4 fitas antropométricas. Quanto maior o número de equipamentos disponíveis, mais dinâmica será a prática, uma vez que menos participantes terão que revezar o uso e menor será o tempo ocioso aguardando os colegas finalizarem suas medições com os equipamentos.

O conteúdo deve ser ministrado de forma agradável e dinâmica, com pouca teoria - focalizada na revisão das técnicas - e muita prática, em que se avaliará a qualidade das medidas dos participantes e se buscará identificar e corrigir os erros de medição. Em sua revisão de literatura, Salas et al. ${ }^{15}$ identificaram que somente $59 \%$ dos estudos publicados acerca de treinamento de equipe mesclaram os métodos instrucionais tradicionais (aula/palestra) com demonstrações, simulações e práticas. A maioria iniciou com palestra em sala de aula, debates ou sessão de vídeo, e em seguida disponibilizou tempo para a prática das novas competências. Segundo os autores, momentos teóricos sempre devem ser alternados com prática de medição a fim de favorecer o aprendizado ${ }^{15}$, assim como evitar desinteresse e cansaço do grupo. Salienta-se que o simples ato de praticar não é suficiente para garantir o aprendizado. As atividades devem ser sempre guiadas pelo instrutor, que deve mostrar a conexão entre conteúdo apresentado e sua incorporação à rotina do profissional ${ }^{15}$, assim como identificar e corrigir problemas relacionados às técnicas de medição. 
Sempre que possível, deve-se buscar praticar as medições em condições similares às da rotina do profissional (exemplo: público-alvo, equipamentos), pois é uma oportunidade de se experimentarem os mesmos processos cognitivos e comportamentais pelos quais passarão em seu trabalho, sem, no entanto, trazerem danos aos indivíduos avaliados, já que se trata de um "ambiente artificial" 15 . É importante lembrar que caso a prática das técnicas seja realizada entre os próprios participantes, particularmente quanto à medida da cintura, deve-se atentar para que participem da capacitação com roupa que permita medição, evitando o uso, pelos participantes, de vestidos, jardineiras ou outras roupas de peça única.

A carga horária poderá ser definida por cada unidade, que variará conforme o número de medidas antropométricas e ciclos de vida a serem contemplados na atividade. Para uma capacitação que contemple até 3 medidas corporais (por exemplo: peso, estatura e perímetro da cintura de adultos), a atividade deve ter, no mínimo, 4 horas de duração, sendo duas horas direcionadas para sensibilização, revisão das técnicas e troca de experiências com a equipe, e duas horas para treinamento, identificação e correção de fragilidades na prática dos profissionais, avaliação da qualidade das medidas e discussão sobre os resultados. Quanto mais medidas antropométricas e ciclos de vida forem incluídos, maior deverá ser a carga horária da capacitação.

É importante ressaltar que de nada adiantará a prática se não for possível avaliar se o profissional realmente está medindo satisfatoriamente ou não. Por isso, sempre ao fim de cada capacitação, deve-se avaliar a qualidade da medição de cada participante, isto é, o grau de precisão e exatidão diante de um "padrão-ouro". Esse profissional, considerado o "padrão-ouro", poderá ser do quadro permanente da unidade ou um especialista convidado para conduzir a capacitação e as atividades práticas, e deve ser um antropometrista bastante criterioso e experiente, que realize medições corporais em sua rotina e participe de treinamentos periódicos. Para verificar se os participantes apresentam um grau de aptidão satisfatório, cada profissional deve medir durante a prática no mínimo 10 indivíduos em duplicata para cada medida antropométrica ${ }^{10}$. A partir dessas mensurações, a precisão e a exatidão poderão ser avaliadas utilizando o método do Erro Técnico de Medição ${ }^{17}$ ou o da Padronização ${ }^{18}$. Ambos os protocolos têm como objetivo determinar quão próximos de um padrão aceitável se encontram as medidas antropométricas e identificar onde os erros estão sendo cometidos para que possam ser eliminados ou, pelo menos, minimizados $^{6}$. É desejável que a unidade mantenha registro de cada uma dessas atividades, assim como da precisão e exatidão de cada participante, favorecendo a avaliação dos profissionais ao longo do tempo.

As capacitações podem ser realizadas fora ou dentro do ambiente de trabalho. Independentemente do local, os participantes devem se comprometer exclusivamente com a capacitação, desligando-se completamente de todas as outras tarefas de seu cotidiano e focalizando-se apenas em seu aperfeiçoamento. Para a parte prática, é importante lembrar que, nos estabelecimentos de saúde, ainda não têm feito parte do projeto físico-funcional salas exclusivas para antropometria, que é realizada na sala de preparo do paciente ${ }^{19}$. Assim, mesmo que a atividade seja realizada dentro da unidade de saúde, recomenda-se que os equipamentos sejam levados para a sala da capacitação, que deve ter tamanho suficiente para posicionar os equipamentos adequadamente e permitir a prática dos participantes sem incômodo por falta de espaço. Dessa maneira, os participantes da capacitação não são interrompidos durante suas medições, e o atendimento de rotina da sala de preparo não é prejudicado.

A última etapa do programa de capacitação é a avaliação, na qual se verifica a efetividade da capacitação em atender as necessidades previamente identificadas. Para a avaliação do retorno do investimento nessa atividade é necessário que seus objetivos sejam bem estabelecidos 
previamente, assim como os indicadores e os mensuradores objetivos, uma vez que, em função deles, os resultados serão avaliados ${ }^{13}$.

Segundo Kraiger et al. ${ }^{20}$, essa avaliação pode ser feita em quatro níveis: reações (exemplo: Os profissionais gostaram da capacitação? Acharam que foi útil?), aprendizagem (exemplo: Os formandos aumentaram sua compreensão acerca das competências específicas de formação?), comportamento (exemplo: Os participantes modificaram seu comportamento no trabalho ou em simulações relevantes?), e resultados ou desfechos (exemplo: Os problemas anteriormente identificados sofreram impacto positivo importante?).

Em antropometria, a avaliação se dá principalmente nos três primeiros níveis, já que modificações nos resultados dificilmente conseguem ser mensuradas (exemplo: Houve redução no percentual de crianças com erro no diagnóstico nutricional por falha durante a medição de peso/ estatura?), pois, na rotina das unidades de saúde, os pacientes são medidos apenas pelo profissional de saúde, e não há "padrão-ouro" para comparar e avaliar se as medições estão adequadas, mas vale a pena ressaltar que, apesar de não mensuradas, essas mudanças se fazem presentes e devem sempre ser perseguidas.

\section{O N CLUSÃ O}

O fomento a programas de formação profissional, com ênfase para a capacitação em antropometria, é uma estratégia de grande valor para subsidiar a geração de informações nutricionais de boa qualidade e fortalecer o SISVAN no país, sistema essencial para formular políticas e desenhos de programas públicos e gerar o retorno necessário para o aperfeiçoamento contínuo das medidas de intervenção em saúde.

Reciclagens periódicas devem integrar de forma permanente a agenda de atividades prioritárias dos serviços de saúde, com ênfase na prática de medições orientada por profissional com pleno conhecimento das técnicas. Programas consisten- tes e bem planejados, concebidos para serem mais do que simples transmissões de informações, são a chave para a criação de um ambiente favorável ao aprendizado e desenvolvimento do profissional, assim como para fortalecer a cultura organizacional e garantir o alcance dos objetivos de promoção, atenção e vigilância em saúde do Sistema Único de Saúde.

\section{COLABORADORES}

Todos os autores participaram de todas as fases do artigo.

\section{REFERÊ NCIAS}

1. World Health Organization. Physical status: the use and interpretation of anthropometry. Geneva: WHO; 1995. WHO Thecnical Report Series, n.854.

2. Ferreira MG, Sichieri R. Antropometria como método de avaliação do estado de nutrição e saúde do adulto. In: Kac G, Sichieri R, Gigante DP, organizadores. Epidemiologia nutricional. Rio de Janeiro: Atheneu; 2007. p.93-104.

3. Brasil. Ministério da Saúde. Política Nacional de Alimentação e Nutrição. Brasília: MS; 2012.

4. Brasil. Ministério da Saúde. Vigilância alimentar e nutricional - Sisvan: orientações básicas para a coleta, processamento, análise de dados e informação em serviços de saúde. Brasília: MS; 2004.

5. Barros DC, organizador. Sisvan: instrumento para o combate aos distúrbios nutricionais na atenção à saúde: a antropometria. Rio de Janeiro: Fiocruz; 2010

6. Brasil. Ministério da Saúde. Secretaria de Atenção à Saúde. Relatório das capacitações macrorregionais em atitude de vigilância: Sistema de Vigilância Alimentar e Nutricional (Sisvan) 2005. Brasília: MS; 2006.

7. Pereira MG. Epidemiologia: teoria e prática. Rio de Janeiro: Guanabara Koogan; 2005.

8. Willett W. Nutrition epidemiology. New York: Oxford University Press; 1990.

9. Bagni UV, Fialho-Júnior CC, Barros DC. Influência do erro técnico de medição em antropometria sobre o diagnóstico nutricional. Nutrire. 2009: 34(3):187-200.

10. Ulijaszek SJ, Kerr DA. Anthropometric measurement error and the assessment of nutritional status. $\mathrm{Br} J$ Nutr. 1999; 82(3):165-77. 
11. Lohman TG, Roche AF, Martorell R. Anthropometric standardization reference manual. Champaign (IL): Human Kinetics Books; 1988.

12. Frainer DES, Adami F, Vasconcelos FAG, Assis MAA, Calvo MCM, Kerpel R. Padronização e confiabilidade das medidas antropométricas para pesquisa populacional. Arch Latinoam Nutr. 2007; 57(4): 335-42.

13. Chiavenato I. Gestão de pessoas. $2^{a}$ ed. Rio de Janeiro: Elsevier; 2004.

14. Ferreira $A B H$. Novo dicionário aurélio da língua portuguesa. $3^{\text {a }}$ ed. Curitiba: Positivo; 2004.

15. Salas E, DiazGranados D, Weaver SJ, King H. Does team training work? Principles for health care. Acad Emerg Med. 2008; 15(11):1002-9.

16. Freire P. Pedagogia do oprimido. Rio de Janeiro: Paz e Terra; 1987.
17. Norton K, Olds T. Antropométrica. Porto Alegre: Artmed; 2005.

18. Habicht JP. Estandarización de metodos epidemiologicos cuantitativos sobre el terreno. Bol Oficina Sanit Panam. 1974; 76(5):375-84.

19. Brasil. Agência Nacional de Vigilância Sanitária. Resolução RDC n 50, de 21 de fevereiro de 2002. Dispõe sobre o Regulamento Técnico para planejamento, programação, elaboração e avaliação de projetos físicos de estabelecimentos assistenciais de saúde. Diário Oficial da União. 200321 set.

20. Kraiger K, Ford JK, Salas E. Application of cognitive, skill-based, and affective theories of learning outcomes to new methods of training evaluation. J Appl Psychol. 1993; 78(2):311-28.

Recebido em: 19/4/2011

Versão final em: 27/3/2012

Aprovado em: 8/5/2012 